\title{
Structure and dynamics of Candida rugosa lipase: the role of organic solvent
}

\begin{abstract}
The effect of organic solvent on the structure and dynamics of proteins was investigated by multiple molecular dynamics simulations (1 ns each) of Candida rugosa lipase in water and in carbon tetrachloride. The choice of solvent had only a minor structural effect. For both solvents the open and the closed conformation of the lipase were near to their experimental X-ray structures ( $\mathrm{C} \alpha$ rms deviation 1-1.3 $\AA$ ). However, the solvents had a highly specific effect on the flexibility of solvent-exposed side chains: polar side chains were more flexible in water, but less flexible in organic solvent. In contrast, hydrophobic residues were more flexible in organic solvent, but less flexible in water. As a major effect solvent changed the dynamics of the lid, a mobile element involved in activation of the lipase, which fluctuated as a rigid body about its average position. While in water the deviations were about $1.6 \AA$, organic solvent reduced flexibility to $0.9 \AA$. This increase rigidity was caused by two salt bridges (Lys85-Asp284, Lys75-Asp79) and a stable hydrogen bond (Lys75-Asn 292) in organic solvent. Thus, organic solvents stabilize the lid but render the side chains in the hydrophobic substrate-binding site more mobile. (c) Springer-Verlag 2004.
\end{abstract}

Keyword: Lid movement; Lipase; Molecular dynamics simulation; Organic solvent 